\title{
Effect of P-alaxin on some neurobehavioural parameters in Swiss white mice
}

\section{O. Nku}

Department of Physiology, Faculty of Basic Medical Sciences, College of Medical Sciences, University of Calabar, Calabar-Nigeria

\section{A. O. Essien}

Department of Physiology, Faculty of Basic Medical Sciences, College of Medical Sciences, University of Calabar, Calabar-Nigeria

A. U. Ime*

Department of Physiology, Faculty of Basic Medical Sciences, College of Medical Sciences, University of Calabar, Calabar-Nigeria

${ }^{*}$ Corresponding author. E-mail: akaninyeneime1@gmail.com

\begin{abstract}
The effect of $P$-alaxin on some neurobehavioural parameters was studied in Swiss white mice. 21 mice were randomly assigned to three groups $(n=7)$ namely; control group, clinical dose group and high dose group. Animals in control group received normal rat chow and standard feed ad libitum. Animals in the clinical dose group were given $\mathrm{P}$-alaxin at a dose of $0.01 \mathrm{mg} / \mathrm{kg}$ body weight while animals in the high dose group were given $\mathrm{P}$-alaxin at a dose of $0.02 \mathrm{mg} / \mathrm{kg}$ body weight. The results obtained showed that, in the open field maze test, the clinical dose and high dose groups showed significant increase $(P<0.05)$ in stretch attend posture compared to control. Conversely, there was a significant decrease $(P<0.05)$ in line crossings of high and clinical dose groups when compared to control. In the light/dark transition box test, the clinical and high dose groups showed a significant decrease $(P<0.05)$ in the frequency of line crossings when compared to control. In the morris water maze test, the high and clinical dose groups showed a significant inncrease in frequency of swimming latency when compared to control group. From the total analysis obtained, P-alaxin decreases locomotion and exploratory activities, it impairs motivation and the tendency to investigate the environment as well as reduction in visuospartial learning and memory.
\end{abstract}

Keywords: P-Alaxin, Dihydroartemisinin, Piperaquine phosphate, Neurobehaviour

\section{Article Info}

DOI: 10.31018/jans.v10i4.1947 Received: November 14, 2018 Revised: November 25, 2018 Accepted: November 29, 2018

\section{How to Cite}

Nku, C.O. et al. (2018).

Effect of P-alaxin on some neurobehavioural parameters in Swiss white mice. Journal of Applied and Natural Science, 10(4): 1308-1313

\section{INTRODUCTION}

Each tablet of P-alaxin contains $40 \mathrm{mg}$ of dihydroartemisinin and $320 \mathrm{mg}$ of Piperaquine phosphate. Dihydroartemisinin is a powerful plasmodicide of malaria parasite gamete which is highly effective against all forms of malaria including multi-drug resistant malaria (Ashley et al., 2005). Dihydroartemisinin is the active metabolite of all artemisinin compounds (artemisinin, artesunate and arthemeter) and is also available as a drug itself. It is a semi synthetic derivative of artemisinin - derived anti-malarial drugs. It is in combination with piperaquine and has been shown to be equivalent to arthemeter/lumefantrine (Cabello et al., 2011). Dihydroartemisinin mainly intervenes with the membrane structures of trophozoitics (erythrocytic asexual forms) causing distended mitochondria, swollen nuclear membranes; dissociation of ribosomes from endoplasmic reticulum leading to cytoplasmic vacuolization and auto phagocytosis (Ashley, 2005). Piperaquine phosphate interferes with physiological function of the food vacuole membrane of the trophozoites leading to autophagocytosis of the parasites (Cabello et al., 2011). $\mathrm{P}$-alaxin has been widely used by humans as medicine for the treatment of Malaria in adults and children with reported side effect such as nausea or vomiting. However, there is paucity of knowledge on its effect on the nervous system particularly some neurobehavioral effects. Hence this study seeks to evaluate its effect on some neurobehavioral parameters.

\section{MATERIALS AND METHODS}

Experimental animals and protocol: Twenty one (21) Swiss white mice with average weight of $25 \mathrm{~g}$ used in this study were purchased from the animal house of the Department of Pharmacology and housed in the animal house of the Department of Physiology, University of Calabar. They were randomly assigned on three groups $(n=7)$ thus; Group 1: Control, group 2: Clinical dose, group 3: high dose. This was done after ethical 
approval was granted by the Animal Ethical Committee of the Faculty of Basic Medical Sciences, University of Calabar.

Before the experiment, the animals were kept in the animal house in ventilated cages under normal temperature and 12/12 lights/dark cycle. Animals were fed with normal ration of rodent chow from vital feeds each morning and water ad libitum. P-alaxin was administered for 3 days before the commencement of the experiment. On each day of experiment, administration was carried out five minutes before commencement of each sequence of neurabavioural experiment. Those in the clinical dose group were given $\mathrm{P}$-alaxin administered doses at a dosage of $0.01 \mathrm{ml}$ via oral route using the small graduated syringe attached with a feeding tube while those in high dose group were given $\mathrm{P}$-alaxin administered doses at a dosage of $0.02 \mathrm{ml}$ via oral route using the small graduated syringe attached with a feeding tube.

Open field maze test: The standard open field test is commonly used to assess locomotor, exploratory and anxiety - like behaviour in laboratory animals (rats/mice) as described by Bisong et al. (2018). This test is particularly useful in evaluating the effects of anxolytic and anxiogenic drugs, locomotor responses to drug and as all behavioural responses to novelty. The open field area generally consists of an empty and bright square (or circular) arena surrounded by walls to prevent animal from escaping. The animal is usually placed in the center of the arena and its behaviour recorded over a chosen period (from 3 to $15 \mathrm{~min})$. The open field task approaches the conflict between the innate fear that rodents have of the central area of a novel or brightly lit open field versus their desire to explore new environments. When anxious; the natural tendency of rodents is to prefer staying closed to the walls (thigmotaxic behaviour).

Behaviours scored included: frequency of line crossings which is the number of times the animal crossed lines drawn on the floor of the open field maze. Walling frequency with which the animal stands on hind legs or leans against a wall of the box with its front paws. Stretch attend postures which is the frequency with which the animal demonstrates forward elongation of head and shoulders followed by retraction to original position.

Light and dark transition box test: The light/ dark transition box apparatus was used to measure some neurobehavioural parameters as described by Bisong et al. (2017) located in a $2 \times 5 \mathrm{~m}$ laboratory room. Rats were carried into "the test room in their home cages and placed in the center of the white chamber facing the opening and were allowed to explore the apparatus for 5 minutes. An observer sitting quietly about $1 \mathrm{~m}$ from the apparatus recorded the behaviour of the animals in the box. A video camcorder located approximately $150 \mathrm{~cm}$ above the center of the maze also recorded the mouse's behaviour. Behaviours were later scored by video play back using Hindsight for MSDOS version 1.5 Computer Software. After 5 minutes, mice were removed from the box by the base of their trails and returned to their home cage. The maze was then with a solution of $70 \%$ ethyl alcohol and permitted to dry between tests. Behaviours scored included: frequency of line crossings which is the number of times the animal crossed lines drawn on the floor of the light and dark sides of the box. Rearing frequency with which the animal stands on hind legs or leans against a wall of the box with its front paws.

Morris water maze test: The Morris water maze has been used extensively to study strain differences in spatial learning in mice and the effects of maze parameters on strain differences in spatial learning (Chapillon and Debouzie, 2000). Considerable use has been made of the Morris water maze for studying spatial learning in transgenic and mutant mice the effects of brain injury on spatial learning and the effects of drugs on spatial learning (Dalm et al., 2000).

During the test period, each mouse was placed in a clean empty cage (no bedding); paper towel was torn and placed in the bottom of the cage to allow the mice to dry more quickly. This paper towel was replaced when it became completely wet. Mice were then run in squads of 5-6 with 10 minutes between each trial for each mouse. Water from external sources like taps, bore holes etc was fetched and poured into a large round basin and powdered milk or chalk was grounded in powder form and poured into the water.

During acquisition training the water was adjusted appropriately such that the platform was covered by $1 \mathrm{~cm}$ of water (invisible platform), the platform was placed in the centre of the North West quadrant. Each animal relieved 4 trials of 60 seconds per day. The starting positions of the animals were predetermined, which prevented any sequence of 2 trials to be repeated by the animal during any other day. Possible start positions were at the boundaries of the quadrants (e.g West, North East or South). Each mouse was removed from its holding cage using a small clean $500 \mathrm{ml}$ plastic yogurt container to minimize handling stress. The animal was when placed into the water at the appropriate start position, facing the center of the pool, the mouse was then permitted to explore the pool and to search for the hidden escape platform for 60 seconds. When the animal couldn't locate the platform during the allotted time, the animal was guided onto the platform using the plastic container. Once on the platform, the mice were permitted to visually explore their surroundings for 20 seconds at which point, the researcher picked up the mouse in the plastic container and returned 
the mouse to the appropriate holding cage. The next mouse was then placed in the pool and the same procedure was followed. Each animal completed 4 trials per day over 3 days making a total of 12 trials of acquisition training.

Reversal training began on day 4 . The invisible platform was moved to the opposite quadrant (Southeast quadrant) and mice were again assigned to appropriate start positions; the same procedures as in acquisition training were again carried out during reversal training. Each of the animals completed 4 trials per day for 3days, a total of 12 trials of reversal training.

A probe trial was conducted on the day 7 . There was no escape platform in the maze at all. Each animal completed one trail of 60 seconds. Each mouse was placed in the maze from one of the four possible start positions and allowed to explore the pool.

The visible platform task was conducted on day 8 . The visible platform was placed in the Southeast quadrant of the pool. The same procedures as in acquisition and reversal training were carried out and mice completed 4 trails.

During acquisition and reversal training the following behaviours were measured;

Swim latency which was the time it took to find and mount the escape platform.

Thigmotaxic movement which is frequency by which the animal attempts to jump or leap out of the size of the circular pool of water.

Proximity movement which is the frequency by which the animal swim to the escape plant form without climbing it.

Statistical analysis: Data collected during the study were analyzed using analysis of variance (ANOVA) and post-hoc student Neuman - Keuls test. Results were presented as mean + SEM.

\section{RESULTS}

Effect of p-alaxin on frequency of rearing in the open field maze: The mean frequency of rearing in the open field maze test for the control group, high dose group and clinical dose group were $3.00 \pm 2.47,3.00 \pm 2.47$ and $5.40 \pm 2.77 / 5$ mins. There was no significant difference between the clinical dose group and the control group. There was also no significant difference between the high dose group and the control group.

Effect of p-alaxin on frequency of walling in the open field maze: The mean frequency of walling in the open field maze test for the control group, high dose group and clinical dose group were $19.80 \pm 3.54,15.40 \pm 3.08,14.80 \pm 0.58 /$ 5 mins. No significant difference existed in the mean values across the groups.

Effect of p-alaxin on frequency of stretch attend posture in the open field maze: The mean frequency of stretch attend posture in the open field maze test for the control group, high dose group and clinical dose group were $6.40 \pm 1.54$, $14.80 \pm 0.58$ and $8.20 \pm 1.32 / 5$ mins. There was a significant increase $(P<0.05)$ between the clinical dose group as well as the high dose group when compared to control group.

Effect of $p$-alaxin on frequency of line crossings in the open field maze: The mean frequency of line crossings in the open field maze test for the control group, high dose group and clinical dose group were $62.80 \pm 4.96,50.80- \pm 4.55,44.20 \pm$ $3.93 / 5$ mins. There was a significant decrease $(P<0.05)$ in the high dose and clinical dose groups when compared to control.

Effect of $p$-alaxin on frequency of rearing in the light and dark transition box: The mean frequency of rearing in the light and dark transition box test for the control group, high dose group and clinical dose group were $29.00 \pm 5.50,28.00$ $\pm 3.48,23.29 \pm 4.36 / 5$ mins. These results presented no significant difference between the clinical dose group and the control group. There was no significant difference across the groups.

Effect of $p$-alaxin on frequency of line crossings in the light and dark transition box: The mean frequency of line crossings in the open field maze test for the control group, high dose group and clinical dose group were $65.82 \pm 4.76,48.70-$ $\pm 3.55,46.20 \pm 7.82 / 5$ mins. There was a significant decrease $(P<0.05)$ in the high dose and clinical dose groups when compared to control.

Effect of $p$-alaxin on frequency of swimming latency during acquisition training days 1-3 in the morris water maze: The mean frequency of swimming latency during acquisition training days in the morris water maze test for the control group, high dose group and clinical dose group were $50.81 \pm 2.37,48.64 \pm 3.52$ and $55.82 \pm 3.07$ for day 1 to day 3 respectively. There was a significant increase $(P<0.05)$ in the high dose and clinical dose groups when compared to control.

Effect of $p$-alaxin on frequency of swimming latency during reversal training days 4-6 in the morris water maze: The mean frequency of swimming latency during reversal training days in the morris water maze test for the control group, high dose group and clinical dose group were 21.43 $\pm 3.99,28.68 \pm 4.45$ and $34.36 \pm 3.29$ for day $4-6$ respectively. There was a significant increase $(P<0.05)$ in the clinical dose group compared to the control group. There is no significant difference between $(\mathrm{P}<0.05)$ the high dose group and the control group.

Effect of $p$-alaxin on frequency of swimming latency during the probe trial day in the morris water maze: The mean frequency of swimming latency during the probe trial day in the morris water maze test for the control group, high dose group and clinical dose group were $1.00 \pm 0.31$, $0.86 \pm 0.34$, and $0.71 \pm 0.36$ respectively. There was no significant difference $(P<0.05)$ across the 
groups.

Effect of p-alaxin on frequency of swimming latency during the visible platform in the morris water maze: The mean frequency of swimming latency during the visible platform day in the morris water maze test for the control group, high dose group and clinical dose group were 16.39 $\pm 3.76,11.46 \pm 0.81,12.04 \pm 3.03$, There was a significant decrease $(\mathrm{P}<0.05)$ in the high dose and clinical dose groups when compared to control group.

\section{DISCUSSION}

This study observes the effect of p-alaxin on some neuro behavioural parameters in swiss albino mice. The neurobehavioural parameters considered in the study include locomotor activities which encompass locomotion and exploratory behaviours. Other parameters investigated in the study were fear and anxiety, learning and memory.

The open field apparatus was used to study locomotion and exploratory behaviours. The light/dark transition boxes were used to study fear and anxiety and the Morris water maze used to study -spatial learning and memory.

The open field test has been used as a test that assesses locomotion behaviour. It provides simultaneous measures of locomotion, exploration as well as anxiety (Chi and Schmitt, 2005). Behaviours such as the number of line crosses and stretch attend postures are used as measures of exploration and anxiety. A high frequency of these behaviours indicates increased locomotion and exploration and or a lower level of anxiety. The frequency of line crosses (number of lines crossed per unit time) measures the horizontal locomotor behaviour. It represents the horizontal distance covered (Chi and Schmitt, 2005). The number of lines crossed following administration of $0.01 \mathrm{mg}$ of p-alaxin showed a significant decrease $(P<0.05)$ from the control group. The high dose group also showed a significant decrease from the control group and recorded a decrease in the number of line crossings compared to the control group but higher than the clinical dose group. The drug showed a reduction in locomotion activity.

The light/dark transition box test was also employed to study locomotion activity. It is a test for locomotion and exploratory behaviour. The frequency of line crosses following administration with p-alaxin showed a significant decrease $(P<0.05)$ when compared to the control group. This trend was also seen in the high dose group. The result of this experiment has shown that $p$ alaxin has depressive or sedative effect as the drug decreased locomotion activity in both the open field and the light/dark transition box.

The effects of treatment with the anti-malarial drug, $p$-alaxin on fear and anxiety related behaviour in mice was studied using the behaviours scored in the open field 5-minutes test and the light/dark transition box test. The high and clinical dose groups recorded an increase in the stretch attend posture compared to control. Stretch attend posture are risk-assessment behaviour which indicate that the animal is hesitant to move from its present location to a new position (Gallagher, 2008) and thus a high frequency of these postures indicates a higher level of anxiety. The hesitation of the animals to move from location to a new position may be due to sedative or depressive effect. The light/dark transition box has been used as a test of unconditioned anxiety and exploratory behaviour. The physiological basis of this test is centred on the conflict between exploring in a novel environment and avoidance of bright light (Bourin and Hascoet, 2003). The light chamber of the light/dark box is the aversive chamber and so, mice would normally spend more time in the dark chamber of the apparatus.

However, an increase in exploratory behaviour in the light chamber (the aversive chamber) of the apparatus indicates decreased fear. This decreased fear (anxiolytic effect) could be caused by anxiolytic drugs/agents (Sandi, 2001).

Table 1. Neurobehavioural Parameters in swiss white mice of all the experimental groups.

\begin{tabular}{|c|c|c|c|}
\hline Parameters & Control & High dose & Clinical dose \\
\hline Frequency of rearing in the open field maze (Per 5min) & $3.00 \pm 2.47$ & $3.00 \pm 2.49$ & $5.40 \pm 2.77$ \\
\hline Frequency of walling in the open field maze (Per $5 \mathrm{~min})$ & $19.80 \pm 3.54$ & $15.40 \pm 3.08$ & $14.80 \pm 0.58$ \\
\hline $\begin{array}{l}\text { Frequency of stretch attend posture in the open field maze (Per } \\
5 \mathrm{~min})\end{array}$ & $6.40 \pm 1.54$ & $14.80 \pm 0.58^{*}$ & $8.20 \pm 1.32^{*}$ \\
\hline Frequency of line crossings in the open field maze (Per $5 \mathrm{~min})$ & $62.80 \pm 4.96$ & $50.80 \pm 4.55^{*}$ & $44.20 \pm 3.93^{*}$ \\
\hline $\begin{array}{l}\text { Frequency of rearing in the light and dark transition box (Per } \\
5 \mathrm{~min})\end{array}$ & $29.00 \pm 5.50$ & $28.00 \pm 3.48$ & $23.29 \pm 4.36$ \\
\hline $\begin{array}{l}\text { Frequency of line crossings in the light and dark transition box } \\
\text { (Per 5min) }\end{array}$ & $65.82 \pm 4.76$ & $48.70 \pm 3.55^{*}$ & $46.20 \pm 7.82^{*}$ \\
\hline $\begin{array}{l}\text { Frequency of swimming latency during acquisition training in } \\
\text { Morris water maze }\end{array}$ & $50.80 \pm 2.37$ & $48.64 \pm 3.52$ & $55.82 \pm 3.07^{*}$ \\
\hline Frequency of swimming latency during reversal training & $21.43 \pm 3.99$ & $28.68 \pm 4.45$ & $34.36 \pm 3.39 *$ \\
\hline Frequency of swimming latency during probe trial & $1.00 \pm 0.31$ & $0.86 \pm 0.34$ & $0.71 \pm 0.36$ \\
\hline Frequency of swimming latency during the visible platform test & $16.39 \pm 3.76$ & $12.04 \pm 3.03^{*}$ & $11.46 \pm 0.81^{*}$ \\
\hline
\end{tabular}


The hidden-platform version of the Morris Water Maze is a test of visuo-spatial learning and memory performance of which is impaired by hippocampal lesion (Chapillon and Debouzie, 2000). The visible-platform version of the Morris Water Maze on the other hand is a non-hippocampal task, which is dependent on the dorsal striatum (Caudate nucleus and putamen) of the basal ganglia (Chapillon and Debouzie, 2000). The visible (cued) platform uses a unique intra maze visual cue that is placed at the location of the escape platform whereas the visuo-spatial learning task uses extra maze cues.

The swim latency was defined as the time it took the mice to locate the hidden platform in the visuo -spatial learning task or the cued platform in the visible platform task, the shorter the swim latency, the better the learning process. Mice which learned faster were able to figure out the spatial location and position of the hidden platform earlier than their counter part, that is, within a very short time. Also, the steeper the gradient of swim latencies in three days acquisition or reversal training, the better the learning. The clinical dose group showed a significant increase in swimming latency for high and clinical dose groups compared to the control group. There was however, a gradual decline in the swim latency during the acquisition to day 3. It appears that the mice in all the groups were able to find the hidden platform at were therefore able to learn equally well during acquisition training. Even though there was significant differences among the groups, the fact is that there was gradual decline in the swim latency compared to control showing that there was some learning in the groups treated with the antimalarial drug as compared to control. Clinically, $p$ alaxin can be taken to improve our memory.

During reversal training, the high dose group showed a significant difference" from the control group, as the high dose group had a higher swimming latency compared to the control group on the sixth day, the clinical dose group maintained a low swimming latency from the fourth to sixth day. Since, shorter latencies mean shorter time to locate the hidden platform and hence better learning ability, the mice treated with the anti-malarial drug had improved learning during reversal training. It can be inferred at this juncture that $p$-alaxin treated mice did better in the swim latency.

Visuo-spatial memory was assessed during the probe trail in the morris-water maze task. During the probe trial, the hidden platform was removed and mice were introduced into the maze and allowed to explore for 60 seconds, during which the quadrant duration (the time spent exploring each quadrant) were recorded. It is expected that mice which have a good memory would spend more time exploring the guardant which had the hidden platform during the reversal training, in this case, the South-east (SE) guardant. Mice treated with $p$ alaxin, showed no significance difference between the clinical dose group and the control group, the high dose group when compared to the control group also showed no significant difference and had a higher swimming latency. There was a significant increase $(P<0.05)$ in the time spent in the SE quadrant. This increase in the SE quadrant duration implies that there was improvement in memory. This is consistent with the learning curve obtained during the reversal training.

The cued version of the Morris-water maze assesses cued learning and visual integrity of the animals tested. Impairment in performance in the hidden platform task may be due to some brain lesions or drugs which may affect the motivation to escape or sensory motor factors rather than spatial learning. This cueing procedure, in which the escape platform protrudes above the water surface, provides a control for this (Morris, 1984). Here also, the swim latencies were used for the comparisons. Shorter swim latencies in the visible platform task indicate improved cued learning. The reverse also holds. Swimming latency during visible platform task of the water maze task, the clinical dose group showed a significant difference to the control group, as the clinical dose group had a higher swimming latency compared to the control group and the high dose group also showed a significant difference $(P<0.05)$ when compared to the control group and having a higher swimming latency than both the clinical dose group and the control group. Administration of $p$ alaxin improved place learning in much the same way as there was improved reversal learning. The results in this study showed that $p$-alaxin improved memory in mice.

From the result obtained on the locomotion and exploratory behaviour in mice, the treatment with the anti-malarial drug, $p$-alaxin decreased both locomotion and exploratory activity. The drug is sedative. The drug impaired motivation. The drug also tends to increase fear and anxiety. Furthermore, results in this study also showed that $p$ alaxin decreases visuo-spartial learning and memory in mice.

\section{Conclusion}

From the results obtained from this study, it can be concluded that malaria treatment with $p$-alaxin decreases locomotion and exploratory activity in swiss mice. P-alaxin also impairs motivation as well as reduction in visuospartial learning and memory. More so, P-alaxin also increases fear and anxiety. If these results are to be applicable to humans, then this calls for concern and caution as consistent use of $p$-alaxin for the treatment of malaria may pose debilitating consequences on humans as seen from the results of this study. 


\section{REFERENCES}

1. Ashley, E. A., McGready, R., Hutagalung, R., Phaiphun, L., Slight, T., Proux, S. and Nosten,F. (2005). A randomized, controlled study of a simple, oncedaily regimen of dihydroartemisinin-piperaquine for the treatment of uncomplicated, multidrug-resistant falciparum malaria. Clinical Infectious Diseases, 41 (4): 425-432.

2. Bisong, S. A., Nku, O., Nwoke, U. and Osim, E. (2018). Crude aqueous leave extract of carica papaya linn (pawpaw) reduced anxiety and fear related behaviour in cd1 mice. European journal of pharmaceutical and medical research. 5(3): 488-493

3. Bisong, S. A., Okon, U. A., Chukwu, J. A. O., Sanya, O. A., Akinnuga, M. A. and Unirere, G. N. (2017). Long term consumption of coconut oil diet increased anxiety related behaviour in CD1 mice. Journal of Complementary and Alternative Medical Research. 2 (1):1-13

4. Bourin, M. H. and Hascoet, M. H (2003). The mouse light/dark box test. European Journal of Pharmacology, 463: 55-65

5. Cabello, C. M., Lamore, S. D., Bair, W. B., Qiao, S., Azimian, S., Lesson, J. L. and Wondrak, G.T. (2012).
The redox antimalarial dihydroartemisinin targets human metastatic melanoma cells but not primary melanocytes with induction of NOXA-dependent apoptosis. Investigational new drugs, 30(4):1289-1301

6. Chapillon, P. F. and Debouzie, A. B. (2000). BALB/C mice are not so bad in the Morris water maze. Behavioural Brain Research, 117: 115-118.

7. Chi, K. J. and Schmitt, D. (2005). Mechanical energy and effective foot mass during impact loading of walking and running. Journal of biomechanics, 38(7): 1387-1395.

8. Dalm, S., Grootendorst, J., De Kloet, E. R. and Oitzl, M. S. (2000). Quantification of swim patterns in the Morris water maze. Behavior Research Methods, Instruments, and Computers, 32(1): 134-139.

9. Gallagher, B.F. (2008). Fear of the unknown. Safer Communities, 7(3): 22-24.

10.Morris, R. B. (1984). Development of procedure for studying spatial learning in the mice. Journal of Neuroscience Method, 11: 47-60.

11.Sandi C. E. (2001). Healing anxiety disorders with glucocorticoids. Proceedings of the National Academy of Sciences, 108(16): 6343-6344. 